\title{
Comparative transcriptome analysis revealed differential gene expression in multiple signaling pathways at flowering in polyploid Brassica rapa
}

\author{
Janeen Braynen ${ }^{1,2}$,, Yan Yang ${ }^{1}$, Jiachen Yuan ${ }^{1}$, Zhengqing Xie², Gangqiang Cao ${ }^{2}$, Xiaochun Wei ${ }^{3}$, \\ Gongyao Shi ${ }^{1,2}$, Xiaowei Zhang ${ }^{3}$, Fang Wei ${ }^{1,2^{*}}$ and Baoming Tian ${ }^{2^{*}}$
}

\begin{abstract}
Background: Polyploidy is widespread in angiosperms and has a significant impact on plant evolution, diversity, and breeding program. However, the changes in the flower development regulatory mechanism in autotetraploid plants remains relatively limited. In this study, RNA-seq analysis was used to investigate changes in signaling pathways at flowering in autotetraploid Brassica rapa.

Results: The study findings showed that the key genes such as $C O, C R Y 2$, and $F T$ which promotes floral formation were down-regulated, whereas floral transition genes FPF1 and FD were up-regulated in autotetraploid B. rapa. The data also demonstrated that the positive regulators GA1 and ELA1 in the gibberellin's biosynthesis pathway were negatively regulated by polyploidy in $B$. rapa. Furthermore, transcriptional factors (TFs) associated with flower development were significantly differentially expressed including the up-regulated CIB1 and AGL18, and the down-regulated AGL15 genes, and by working together such genes affected the expression of the down-stream flowering regulator FLOWERING LOCUS T in polyploid B. rapa. Compared with that in diploids autotetrapoid plants consist of differential expression within the signaling transduction pathway, with 13 TIFY gens up-regulated and 17 genes related to auxin pathway down-regulated.

Conclusion: Therefore, polyploidy is more likely to integrate multiple signaling pathways to influence flowering in B. rapa after polyploidization. In general, the present results shed new light on our global understanding of flowering regulation in polyploid plants during breeding program.
\end{abstract}

Keywords: Polyploidy, Transcriptome, Flowering, Regulatory pathway, Transcriptional factors, Brassica rapa

\footnotetext{
*Correspondence: fangwei@zzu.edu.cn; tianbm@zzu.edu.cn

1 School of Life Sciences, Zhengzhou University, Zhengzhou 450001,

Henan, China

${ }^{2}$ Henan International Joint Laboratory of Crop Gene Resources and Improvements, School of Agricultural Sciences, Zhengzhou University, Zhengzhou 450001, Henan, China

Full list of author information is available at the end of the article
}

\section{Background}

The biological and genetic advantages of polyploids relative to diploids are vast, and most studies conclude that polyploids often possess novel traits that are not present in the diploid progenitors $[1,2]$. These novel traits, such as the increase in organ size and biomass, resistance to pests and changes in flowering time could allow polyploids to enter new environmental niches [3-5].

The effects of polyploidization on plant development have been extensively studied at the transcriptome level

c) The Author(s) 2021. This article is licensed under a Creative Commons Attribution 4.0 International License, which permits use, sharing, adaptation, distribution and reproduction in any medium or format, as long as you give appropriate credit to the original author(s) and the source, provide a link to the Creative Commons licence, and indicate if changes were made. The images or other third party material in this article are included in the article's Creative Commons licence, unless indicated otherwise in a credit line to the material. If material is not included in the article's Creative Commons licence and your intended use is not permitted by statutory regulation or exceeds the permitted use, you will need to obtain permission directly from the copyright holder. To view a copy of this licence, visit http://creativeco mmons.org/licenses/by/4.0/. The Creative Commons Public Domain Dedication waiver (http://creativecommons.org/publicdomain/ zero/1.0/) applies to the data made available in this article, unless otherwise stated in a credit line to the data. 
[6, 7]. Gene expression may be significantly altered as ploidy level changes, and thus contributed to changes in phenotypic traits. For example, autotetraploids in Betula platyphylla exhibited increased breast-height diameter, volume, leaf, fruit, and stomata size in autotetraploid plants. Further analysis of this study revealed that such increases in morphological traits might have been contributed by the significant up-regulation of indole acetic acid (IAA) and ethylene signaling transduction hormones [8]. Additionally, in apple plants (Malusdomestica) a dwarf phenotype was observed for synthetic autotetraploid plants. It was indicated that hormones including IAA and brassinosteroid (BR) were also significantly decreased; which indicated a partial interruption of the IAA and BR signal transduction pathways in the polyploidy apple plants [9]. Furthermore, studies have indicated genomic instabilities that occurred during meiosis in some plants might also play some roles in genetic diversities [10]. These genome instabilities occurring during meiosis usually affect neopolyploids [11]. The process by which neopolyploids survived with low diversification and reproductive disadvantages including flowering is still poorly studied [7].

Over recent years, various scientific progress has been made to decipher the molecular basis of vegetative to reproductive transition in polyploid plants [12]. Most alternation during flowering are related to epigenetic and genetic mechanisms. Flowering might be regulated by a complex network of genetic pathways, which were strongly responsive to endogenous changes and environmental stimuli [13]. In allotetraploid Brassica and Arabidopsis plants, plants with delayed flowering from a late and early flowering parent were mostly observed [14]. In addition, the flowering and bolting times for autotetraploid were delayed by 8.3 and 11.2 days compared with the wild type plants [15]. Furthermore, the bolting time and rosette growth in Arabidopsis ploidy plants were remarkably delayed at 22,23 and 26 days in tetraploid, hexaploid and octoploid, respectively [16]. These data implied that changes in flowering variation might be related to subtle alternation in specific signaling or genetic pathways after the induction of polyploidization. Moreover, flowering date and height in alfalfa autotetraploid were associated with CONTANS-like genes [17]. However, with the numerous studies concerning flower development in autotetraploid plants, to best of the authors knowledge, little information is available about the cascade of regulatory pathways and interaction of genes related to the delay in flowering after polyploidization.

In the present study, transcriptome analysis was used to investigate the genome-wide changes of gene expression regulation on flowering in autotetraploid $B$. rapa in comparison with its diploid counterparts, which would further deepen our understanding of polyploidyassociated effects on flowering regulation.

\section{Results}

Phenotypic characteristics in autotetraploid B. rapa

As shown in Fig. 1, the induced polyploid B. rapa plants consisted of significantly enlarged flowers (Fig. 1b, d), when compared to its diploid progenitors (Fig. 1a, c). The chromosome counts for autotetraploid B. rapa plants displayed 40 chromosomes after genome duplication indicating a level of tetraploidy (Fig. 1f) whereas diploids plants consist of a somatic chromosome set of 20 (Fig. 1e). In addition, flow cytometric results indicated a two-fold increase in DNA content in autotetraploid (Fig. 1h), compared with that of DNA content observed for the diploids (Fig. 1g). Moreover, floral transition in the induce plants was delayed by 7-10 days in comparison with the diploids (Fig. 11). The results obtained here demonstrate the induced tetraploid $B$. rapa had enlarged flower organs and postponed flowering after polyploidization (Fig. 1k, i).

\section{Analysis of differentially expressed genes related to flowering}

As both diploid and tetraploid $B$. rapa have the same number of chromosome set, we predicted that the changes in flower development of the tetraploid $B$. rapa may be associated with altered regulatory pathways. To examine genes expression profiles involved in flower development after polyploidization, cDNA libraries were constructed. RNA-seq analysis revealed that 4601 genes consisting of a probability cut of $\leq 0.05$ to be significantly differentially expressed. Functional analysis was performed to identify KEGG pathways affected after polyploidization. KEGG pathway analysis was firstly performed for all DEGs. The results showed approximately $35 \%$ DEGs were assigned to 50 biological pathways with plant hormone signaling transduction pathway (69) enriched with the largest number of DEGs. Biosynthesis of amino acids (48), and protein processing endoplasmic reticulum pathways (47) were also overrepresented with DEGs (Fig. 2a). In addition, flower time-related pathways were also assets to investigate the delay in flowering observed in the autotetraploid plants. Interestingly, all flower-time pathways consisted of differentially expressed genes except the vernalization pathway which was relatively stable. These results indicated that multiple pathways associated with flower development were substantially altered in autotetraploid B. rapa. 

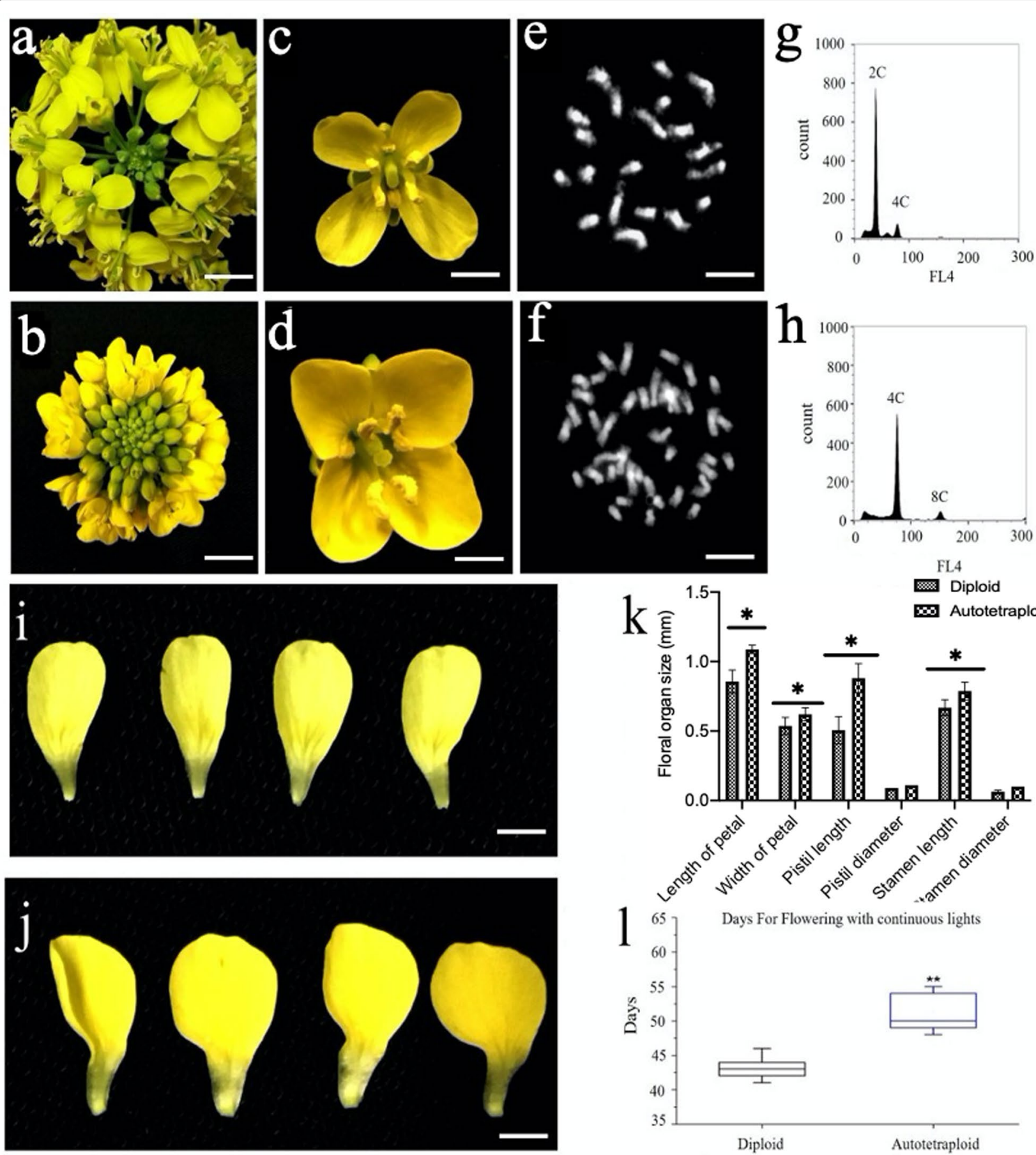

Fig. 1 Phenotypic analysis, chromosome number and flow cytometric analysis of the colchicine-induced B. rapa plants. a, b flower of diploid $B$. rapa and the enlarged flower of B. rapa plant. Bar $=1 \mathrm{~cm} . \mathbf{c}$, d Open flower of the diploid and autotetraploid B. rapa. e, $\mathbf{f}$ Exactly 20 chromosomes in diploid B. rapa and 40 chromosomes counted in autotetraploid B. rapa. Bar $=10 \mu \mathrm{m} . \mathbf{g}, \mathbf{h}$ Peak distribution of nuclear DNA content in autotetraploid in comparison with diploid B. rapa. $\mathbf{i}$, $\mathbf{j}$ differences in petal sizes between diploid and autotetraploid B. rapa. $\mathbf{k}$ Measurements of floral tissues between diploid and autotetraploid. I Depiction of floral transition between the diploid and autotetraploid. Asterisks indicate the following criteria of significances: * $\mathrm{p} \leq 0.05$ 


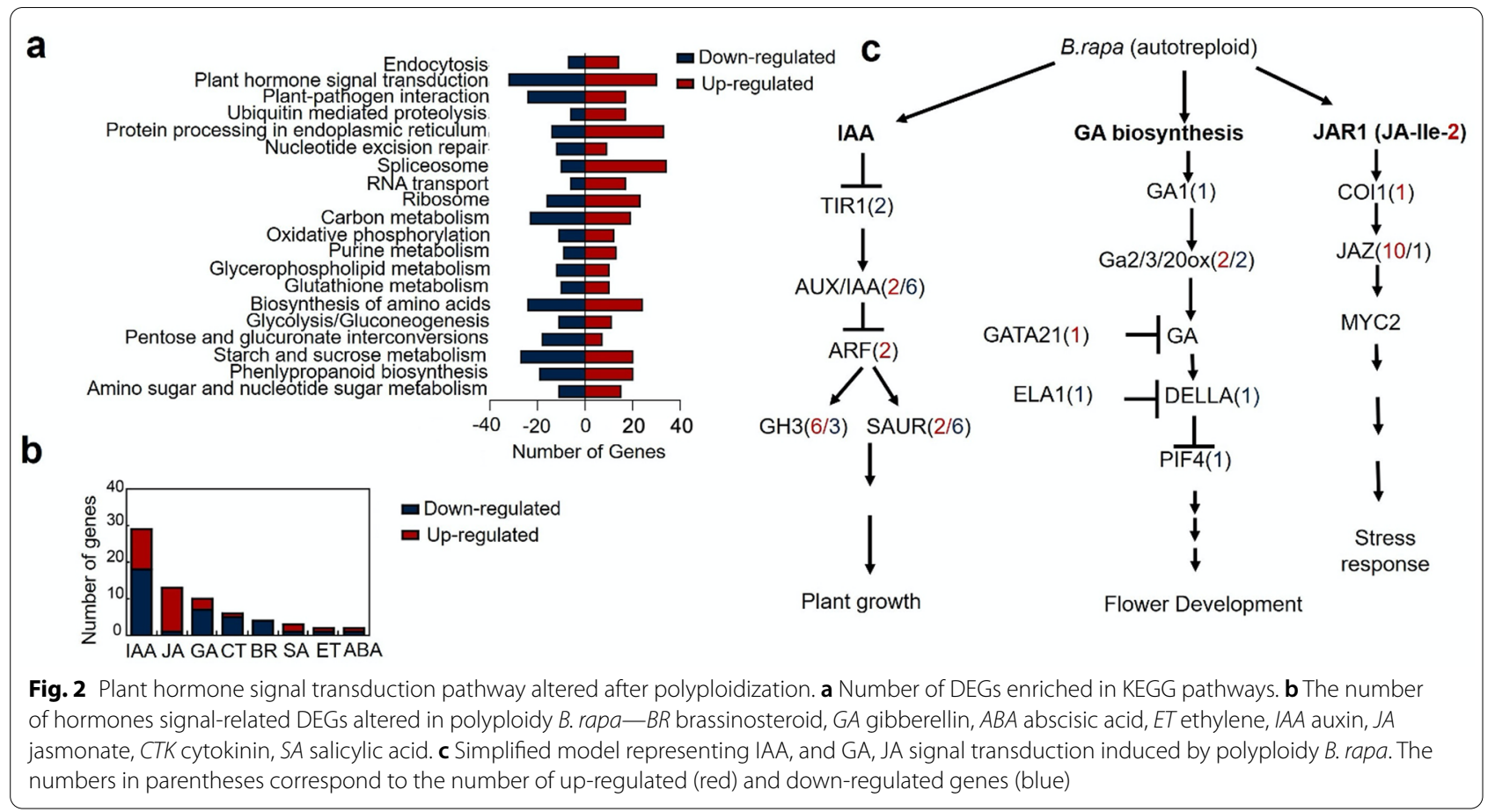

\section{Hormone signaling pathways differentially regulated in polyploid $B$. rapa}

KEGG enrichment analysis showed that 69 DEGs involved in plant hormone signaling transduction pathways were altered in the polyploidy plants (Fig. 2b, Additional file 1: Table S1). More specifically, 42\% (29) were associated with auxin signaling, $18.8 \%$ (13) with jasmonate (JA), 14.5\% (10) with gibberellin signaling (GA), 8.7\% (6) with cytokinin (CTK), 5.8\% (4) with Brassinosteroid (BR), 4.3\% (3) with salicylic acid (SA), 2.9\% (2) with abscisic acid (ABA) and 2.9\% (2) with ethylene (ETHY) signaling (Fig. 2b). Genes enriched in the auxin pathway included 9 GH3 genes, 2 auxin response factors $(A R F)$ genes, 2 GRR-1/TIR1 genes, 8 genes that were downstream response to $A U X / I A A$ and $8 S A U R$ genes were differentially regulated for this pathway (Fig. 2c). From the 29 genes regulated in this pathway, 12 genes were up-regulated and 17 down-regulated. In the JA signaling pathway, two upregulated genes encoding JAR1 protein, 10 up-regulated genes encoding TIFY proteins and one gene up-regulated which encoding Coronatine-insensitive protein 1 COI1 were significantly altered after polyploidization. Enrichment analysis revealed that GA signaling genes DELLA (RGL2) and PIF4, were both down-regulated. In addition, RNA-seq data showed that 8 genes involved in GA biosynthesis were differentially expressed in polyploid $B$. rapa, which included GA1, GA2ox7, GA20ox5, GA3ox4, GA2ox8, CARBON
METABOLISM INVOLVED (GNC), GNC-like (GNLI), CYTOCHROME P450 714A1 (ELA1). Elevated expression of GA20ox5, GNCLI, and GA3ox4 was up-regulated in polyploid $B$. rapa, whereas genes GA2ox7, GA1, GA2ox4, ELA1 were down-regulated.

Further, after polyploidization, the CTK pathway consisted of five down-regulated genes in the Arabidopsis response regulators Family such as $A R R 2, A R R 4, A R R 6$, and two ARR5 and one up-regulated gene (ARR8). Meanwhile, BR signaling had four down-regulated genes including SERK2, BIN2, BIL1 and Protein kinase-related gene. SA signaling consists of two up-regulated NPR genes including $N P R$ and NPR6/BOP2. In addition, PRP1 was down-regulated in this pathway. For the process of ABA signaling pathway, $A B 15$ and $S n R K 2$ were downand up-regulated, respectively. While three PP2C genes were all up-regulated. Furthermore, for ETHY signaling EIN3, CTRI and ERS2 were down- and up-regulated, respectively. These results indicated that polyploidization (chromosome doubling) could lead to a distinct hormone signaling regulation, which may play an important role in the flower development or floral organ growth process in autotetraploid B. rapa.

\section{Genes related to floral organ development}

To further investigate the change in the flower enlargement of autotetraploid plants, all genes related to organ size were assessed. Genes such as GROWTH-REGULATING FACTORS (GRFs) and GRF-INTERACTING 
FACTORs (GIFs), AUXIN-REGULATED GENE INVOLVED IN ORGAN SIZE (ARGOS) and AP2/ERF type transcription factors AINTEGUMENTA (ANT) were not differentially expressed. However, one known gene in Arabidopsis which promote floral organ growth KLU/CYP78A5 was differentially expressed. After polyploidization, two KLU/CYP78A5 genes were up-regulated (Additional file 1: Table S2). These results indicate that the change in organ size may not have occurred due to late floral phenotype observed. However, the floral enlargement occurred may occur due to polyploidization.

\section{Identified unigenes related to flower-time regulation in $B$. rapa}

In addition to changes in autotetraploid B. rapa floral growth, the phenotypic analysis indicated a delay in floral transitioning. Thus, to study the probable cause for the delay flowering observed in autotetraploid B. rapa, flowering related pathways were analyzed including photoperiod/circadian clock pathway, meristem response and development, and vernalization and autonomous pathways. Using homologs from A. thaliana, as a query, 348 genes including 70 transcriptional factors were identified to be involved in flowering specific pathways in $B$. rapa (Additional file 1: Table S3, Additional file 2: Figure S1). Interestingly, among the 348 genes, 52 genes were differentially expressed. Our data indicated that 34.6\% (18 genes) of the 52 DEGs related to flowering were concentrated in the photoperiod/circadian clock pathway (Fig. 3a, Additional file 1: Table S4). Among these, 12 flowering-related genes were significantly down-regulated including CYCLING DOF FACTOR 2 (CDF2), NUCLEAR TRANSCRIPTIONAL FACTOR Y SUBUNIT C-9 (NFYC3), SUPPRESSOR OF OVEREXPRESSION OF CO1 (SOC1) a transcriptional activator of flowering control, APETALA 1A (AP1), EARLY FLOWERING 3 (ELF3), TEMPRANILLO 1 (TEM1), CRYPTOCHROME 2 (CRY2), GLYCINE-RICH RNA -BINDING (CCR1), ZINC FINGER PROTEIN CONSTANS LIKE 9 (COL 9), CHLOROPLAST STEM-LOOP BINDING PROTEIN (CRB), SUPPRESSOR OF PHYA 105 (SPA1), SPA1 RELATED 3 (SPA3), and LHY/CCA1-like

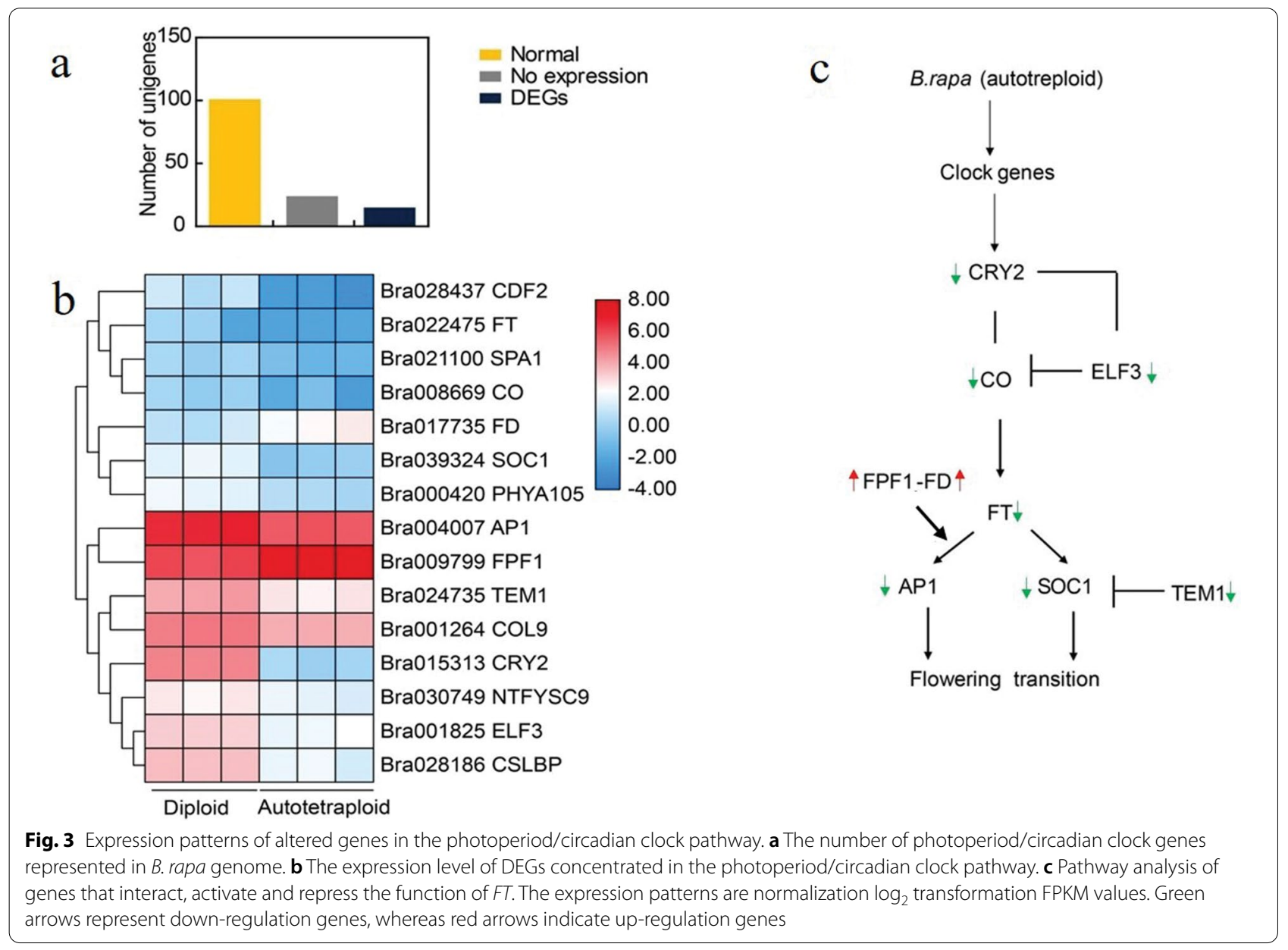


1 genes. In addition, FLOWER PROMOTING FACTOR 1 (FPF1), FLOWERING LOCUS D (FD), EARLY PHTOCHROME RESPONSIVE1 (EPR1) and TWIN SISTER FT (TSF) were significantly up-regulated in autotetraploid B. rapa (Fig. 3b). Furthermore, the initiator of flowering FLOWER LOCUS FT (FT, Bra022475) was also down-regulated (Fig. 3c). Thus, such results imply that the altered expression of photoperiodic/ circadian genes was distinct after polyploidization, indicating a probable cause of delay floral transiting.

For the vernalization and autonomous pathway, only four genes were differentially expressed including FRIGIDA like (FRL) and RNA -BINDING protein (AtCRP7) was found down-regulation in B. rapa. Many genes involved in the vernalization pathway, EMBRYONIC FLOWER 2 (EMF2), VERNALIZATION 2 (VRN2), and FRIGIDA (FRI), were identified to be normally expressed between the autotetraploid and diploid B. rapa. However, FLOWERING LOCUS C (FLC) an important player of the vernalization pathway consists of four genes in B. rapa, but only FLC5 was differentially expressed, up-regulated. These results imply that the vernalization and autonomous pathways were not affected by the change in ploidy level. However, the up-regulation of $F L C 5$ a known floral repressor in B. rapa may have contributed to the delay in flowering.

\section{Expression of transcription factors affected in polyploid $B$. rapa}

To further understand the delay in flowering of autotetraploid $B$. rapa, we identified the transcriptional factors (TFs) that were associated with flower development and floral transition. Over 135 TFs were differentially expressed with $21 \mathrm{TFs}$ associated with flowering; including 12 down-regulated and 9 up-regulated genes between autotetraploid and diploids B. rapa (Fig. 4a, Additional file 1: Table S5). For flowering, transcriptional factors bHLH families were the most represented group of differentially expressed genes consisting of ten genes (Fig. 4b, Additional file 1: Table S5). For bHLH, 3 TFs were

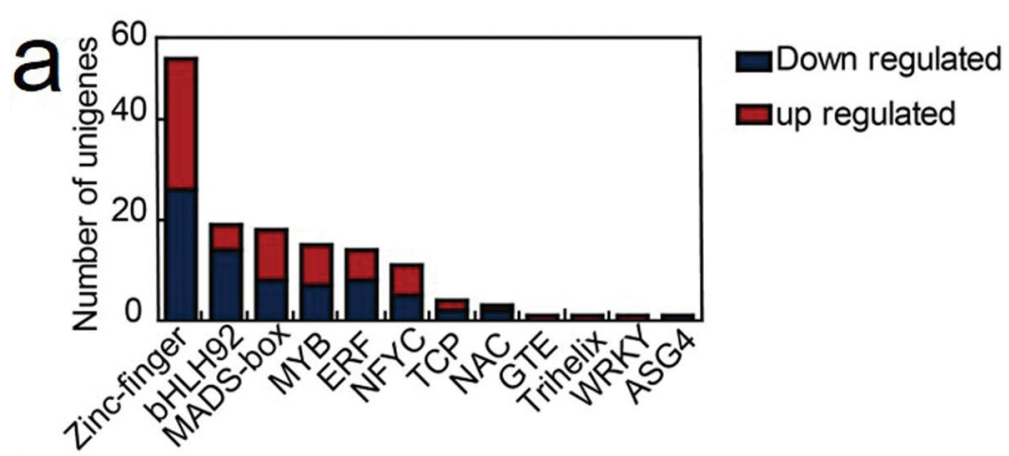

$\mathrm{b}$

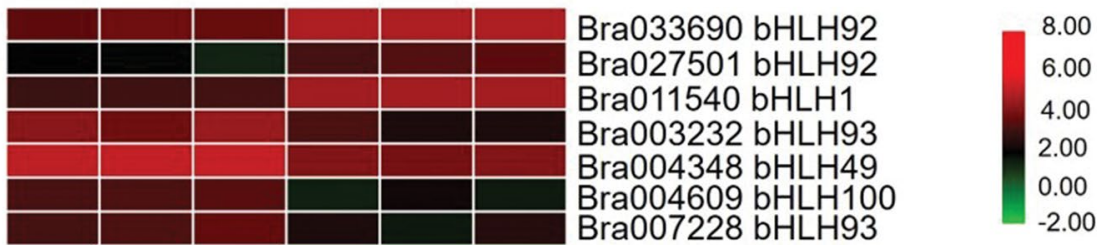

Bra007863 bHLH66

Bra032111 bHLH66

Bra016299 bHLH135

Bra015372 WRKY7

Bra005751 ASG4

Bra014628 AGL18

Bra019018 AGL18

Bra004117 AGL15

Bra000392 AGL6

Bra013304 AGL6

Bra016090 TCP22

Bra0272834 TCP4

Bra039096 TCP20

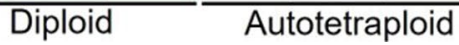

Fig. 4 Differential expressed transcriptional factors (TF) in autotetraploid B. rapa. a Number of transcriptional factors related to flower-time transitioning. $\mathbf{b}$ Heatmap representing TFs related to flowering time 
up-regulated, two $b H L H 92$ and $C I B 1 / b H L H 1$.Whereas, 7 TFs displayed down-regulation including two $b H L H 93$, two bHLH66, bHLH49, bHLH1OO and bHLH135. In addition, MADS-Box TFs two AGL18 and AGL17 were down-regulated, whereas AGL15, AGL19, AGL6 were upregulated. Transcription Factors TCP class I/II consisted of 4 genes, two up-regulated genes TCP2 and two downregulated genes TCP22 and TCP4. Furthermore, WRKY TFs only had one gene which was affected after polyploidization, including WRKY7 (up-regulated) (Fig. 4b). Taken together, these results indicated that transcriptional factors associated with flowering were also affected after polyploidization in B. rapa. In general, a working model was proposed to reveal the changes in regulation pathways at flowering in autotetraploid B. rapa (Fig. 5).

\section{Verification of RNA-seq by qRT-PCR analysis}

To verify the RNA-seq data, nine DEGs were selected for qRT-PCR analysis. The results showed that seven out the nine DEGs displayed similar expressions to the RNA-seq data in both diploid and autotetraploid (Fig. 6; Additional file 2: Figure S2). AGL15 showed dissimilar expression patterns with the RNA-seq data. AGL15 was highly upregulated after qRT-PCR analysis, whereas the expression was decreased for the RNA-seq results. Despite the dissimilar expression displayed for AGL15, these results indicated that the RNA-seq data were reliable and the results were highly reproducible.

\section{Discussion}

Numerous studies on the flower formation of A. thaliana and other model species uncovered several critical regulators for flower development. The polyploid induction results in enlargement of floral organs and in most studies delay flowering for majority of polyploid plants [16]. However, to date most researches have not indicated specific signaling pathways which might affect the observed flowering delay and enlargement in polyploid plants. In this study, the polyploidy-induced changes at flowering in tetraploid B. rapa and changes in signaling transduction pathways were investigated using RNA-seq technologies.

\section{DEGs changes in multiple signal transduction pathway affect floral transitioning in autotetraploid $B$. rapa}

Numerous plant hormones have been shown to participate in transition of flowering including JA, GA, auxin, $\mathrm{ABA}$ and cytokinin $[18,19]$. For floral transition, the role of GA pathway has been thoroughly investigated [20-22]. GA exerts its biological functions on floral transition by degrading DELLA proteins and activating the key floral transition integrators (SOC1, AGL24, and $L F Y$ ) [16]. In A. thaliana, mutation in GA1 locus displayed a severe delay in flowering [23]. After polyploidization in B. rapa, the GA1, DELLA (RGL2) and PIF3 genes were severely down-regulated in autotetraploid $B$. rapa. Moreover, the up-regulation of ABA associated genes PP2Cs block the generation of some $S n R K 2$ genes causing different growth periods between two $B$. rapa accessions (Jin Wawa and Xiao Baojian), during continuous vernalization periods [24]. However, in the two plants used in this study, autotetraploid $B$. rapa exhibited an up-regulation for $S n R K 2$ after polyploidization compared to diploid $B$. rapa. Additionally, the PP2C genes were up-regulated in this study. Plants hormones are known to interact with flowering time genes to effect flowering in majority of plants. JA receptor gene COI1 negatively regulates flowering through repressing the expression of $F T$ [25]. In polyploid $B$. rapa COI1 gene was up-regulated, it is probable that the up-regulation of COI1 may repress the expression of FT. ABA and DELLAs both interact with $F L C s$ to cause alternation during flowering. Therefore, $\mathrm{JA}, \mathrm{ABA}$, and GA signaling may participate in regulating the floral transitioning in autotetraploid B. rapa.

In most flowering plants, the timing of flowering is primarily influenced by numerous flowering time genes. Cryptochrome 2 one of many positive integrators of flowering time, interacts with $C O$ protein to enhance its stability. While $C O / F T$ expression is a core link between the photoperiod induce pathway [26]. In B. rapa, plants under high ambient temperatures consist of reduce expression of $F T$ and delay flowering via a mechanism associated with Histone variant (H2A.Z). The data here indicated that the expression of $F T$ was low but not significant, while $C R Y 2$ was significantly down-regulated with $C O$ and $S O C 1$ displaying stable and down-regulated expressions, respectively. Both $F T$ and $S O C 1$ are known to strongly initiate flowering, thus the differential regulation of such genes could alter flowering in plants. Furthermore, the regulation FT interacts with AP1. The expression of $A P 1$ after polyploidization was down-regulated. The common AP1 down-regulation in floral meristems is commonly associated with late flowering [27]. Moreover, $F L C$ a central player in the vernalization pathway consists of four copies in the $B$. rapa genome [28]. Unlike, $F L C 1, F L C 3$, and $F L C 2$ which are known to delay flowering in B. rapa, FLC5 is a relatively weak regulator in $B$. rapa. For this study, FLC5 was slightly up-regulated but not significant. The expression of $F L C$ is positively regulated by $F R I$ an causative genes in the vernalization pathway [29]. Between the diploid and polyploid plants the FRIGIDA gene were not differential expressed but FRIGIDA-like genes in polyploid $B$. rapa were differentially expressed. Flowering time in $B$. rapa is an complex mechanism due to duplicated genes. Our results suggest the relationship between flowering time pathways and plant hormones signaling trandcution pathways may 


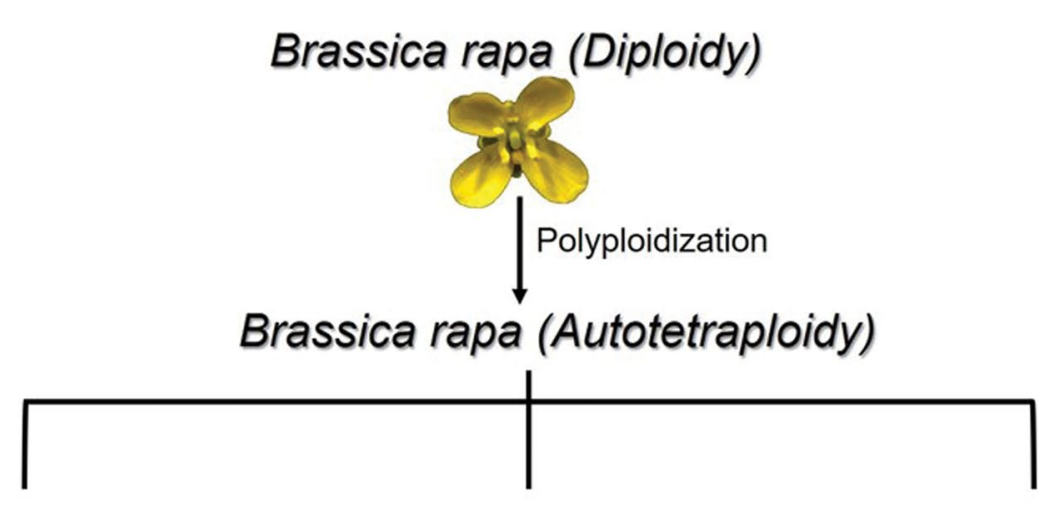

\section{Plant hormone signaling transduction Pathway}

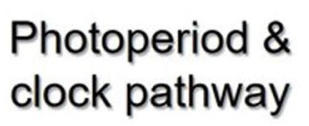

Vernalization

Pathway



Fig. 5 Proposed working model of the flowering cascade observed in autotetraploid B. rapa. Blue representing down-regulated genes while red represents up-regulated genes

contribute to the delay in flowering observed. However, genetic interaction between specific pathways is complex to understand in autotetraploid B. rapa.
Effects of TFs on flowering time variation in autotetraploid B. rapa

MADS-domain transcription factors may positively and negatively regulate the vegetative to reproduction transition in plants. When AGL15 and AGL18 were over-expressed, it significantly reduced the expression 


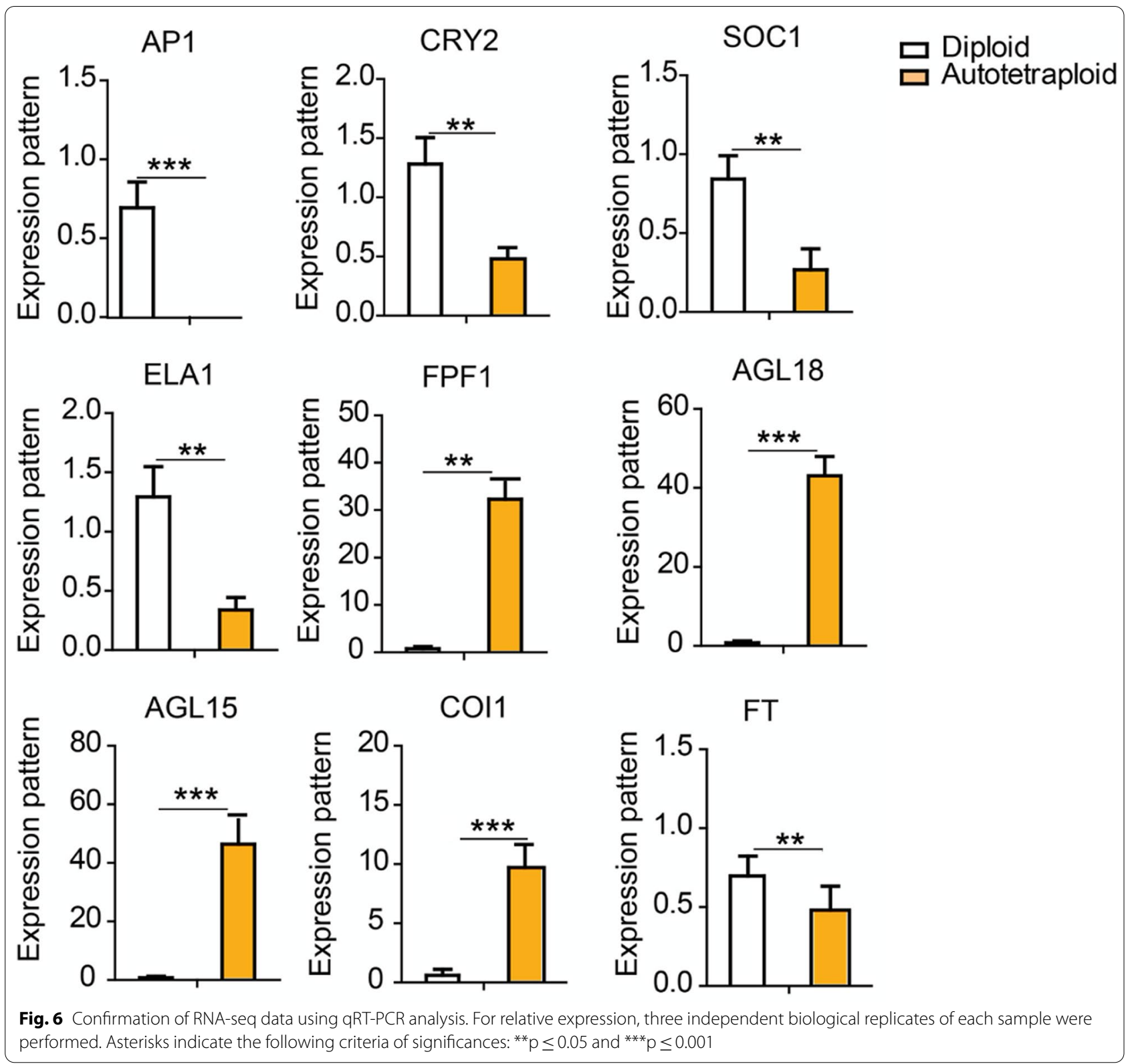

of $F T$ and participated in the late development of flowers. AGL15 and AGL18 were down-and up-regulated, respectively. The results presented here were inconsistent with the previous research which indicated that the expression of $A G L 15$ in most cases consisted of similar expression with $A G L 18$ [30]. These results suggest that after polyploidization, both AGL18 and AGL15 displayed independent expression patterns despite the overlapping functions shared between the MADS-box genes [31]. The high expression of AGL18 may have interact with the expression of FT independent of AGL15 expression. Additionally, one previous study indicated the overexpression of CIB1 caused early flowering in A. thaliana plants [32]. CIB1 a transcriptional factor $b H L H 1$ interacts with $C R Y 2$ to regulate and activate the expression of FT but independent of each other. In this study, the expression of $C R Y 2$ was significantly down-regulated and CIB1 was up-regulated. Most transcriptional factors outline in this study had an essential role in the regulation of polyploid B. rapa flowering variation.

\section{The probable cause of floral growth in autotetraploid $B$. rapa}

Flower size involves the timing of cell proliferation arrest within developing floral organ primordia [33]. The control of such mechanism can involve plant hormones 
[33-38] and genes related to organ growth [39]. Pathways such as Auxin, Brassinosteriod, cytokinins and ethylene, when regulated contribute to numerous functions including, cell proliferation and organ growth. However, in this study, majority of genes related to floral size such as AUXIN-REGULATED GENE INVOLVED IN ORGAN SIZE (ARGOS) or the gene encoding the AP2/ERF type transcription factor AINTEGUMENTA (ANT) and cytokinin oxidase/dehydrogenase $C K X 3$ and $C K X 5$ consist of normal expressions. The only noticeable gene TIFY/JAZ consists of an elevated expression in autotetraploid $B$. rapa. In previous studies overexpression of specific $J A Z$ genes promotes an increase in grain size and increase floret numbers in plants placed under stressful conditions [40]. Additionally, our study indicated that after polyploidization, two KLU/CYP78A5 genes were elevated. An earlier study in Arabidopsis indicated that mutant and overexpressed lines consist of smaller and larger floral organs, respectively [41, 42]. The result indicates that, upon polyploidization, the KLU/CYP78A5 genes may affect floral size, as well as lack genetic interaction from known floral size contributors, including auxin and Brassinosteriod.

\section{Conclusion}

In conclusion, to date studies on the mechanism related to flowering and floral organ in autotetraploid plants focus only on the comparison between the parental lines and polyploids at the phenotypic level. Polyploid are advantageous and considered more favorable than their diploid counterparts; thus, its necessary to clarity its effects on flower development. In this study, autotetraploid and diploid plants were investigated. For comparison purposes it was indicated that autotetraploid and diploids consist of various changes in flowering and floral size, which is similar to most polyploid plants. To decipher the changes in gene expression comparative transcriptome analysis was investigated. According to the results, polyploid $B$. rapa accessions exhibited significant differential regulation in multiple signaling transduction pathways. In comparing the two samples sets, keys genes within the plant hormone signal transduction pathway and flowering time pathway were notably different and the differences indicated a down-regulation for most genes and up-regulation of some for the polyploid plants. For transcription factors, TCP4, bHLH and, WRKY genes exhibited different expression patterns, but these different require further experimental analysis to be confirmed. Notably, KLU/CYP7845, which play a role in floral organ size, exhibited elevated expression patterns after polyploidization in $B$. rapa, which may be a new avenue for research on polyploid $B$. rapa in the future. The comparative analysis of polyploid $B$. rapa at flowering was investigated and summarized in this research, which shed light and provides a foundation for future investigation at the molecular level on the mechanisms contributing to changes in flowering of autotetraploid plants.

\section{Methods \\ Plant materials}

Autotetraploid B. rapa plants were generated artificially by colchicine treatment $(0.1 \% \mathrm{w} / \mathrm{v})$ as previously described [10]. The somatic ploidy level for all polyploid plants used in this experiment was confirmed using flow cytometry [43]. All B. rapa plants used in this experiment were grown in a preferred greenhouse condition of $16 \mathrm{~h} \mathrm{light} / 8 \mathrm{~h}$ dark cycle at temperatures of $22{ }^{\circ} \mathrm{C}$ daytime $/ 18{ }^{\circ} \mathrm{C}$ nights. At the flowering stage, tissue samples were harvested and immediately stored in liquid nitrogen in three biological replicates for further analysis.

\section{Ploidy and morphological analyses of autotetraploid $B$. rapa}

Floral buds in size from 0.8 to $1 \mathrm{~mm}$ were collected and fixed with Carnoy's solution (ethanol: acetic acid $=3: 1$ $\mathrm{v} / \mathrm{v}$ ) for $24 \mathrm{~h}$ at room temperature. The samples were then stored in $70 \%$ ethanol at $4{ }^{\circ} \mathrm{C}$ until use. Anthers were removed from the floret using forceps and a dissecting needle under a stereo-microscope and incubated as previously outlined [44]. The chromosome spreads were prepared as previously described [45]. Chromosome numbers for each sample were visualized using an Olympus BX53 epifluorescence microscope equipped with a cooled CCD DP73 digital camera (Japan Olympus-life science). The floral tissues for phenotypic analysis were harvested upon opening to examined variations among plants with different ploidy levels. To assess bolting and flowering time, we measured (100 plants for each diploid and autotetraploid) the time of induction of bolting until the production of siliques.

\section{Total RNA extraction and mRNA sequencing}

Floral buds with indefinite inflorescence were harvested from each plant in three biological replicates and stored in liquid nitrogen. Sample sets for each genotype were finely ground in liquid nitrogen and total RNA extracted using a modified TriZol (Invitrogen) reagent method with on-column DNase treatment. The poly A + RNA samples collected were annealed, reverse transcribed and ligated to adapters which were complementary to sequencing primers. Further library construction was conducted by the Biomaker Institute (Beijing, China), which included the following procedures PCR amplification and construction of libraries for RNA-seq. The RNA libraries were sequenced on Illumina HiSeq 2000 (Illumina, San 
Diego, California USA). For subsequent analysis, the raw data were processed by base calling and stored in FASTQ files. The generated FASTQ files were deposited in the NCBI Sequence Read Archives (SRA) (https://www.ncbi. nlm.nih.gov/sra) with the accession number SRP104015.

\section{Data analysis: quality control and mapping of raw reads} For data analysis, the FASTQ sequences files were filtered and trimmed similar to procedures as previously outlined [10]. The clean reads were aligned to the reference's genome of $B$. rapa (BRAD http://brassicadb.org/ v.1.5) using the sequence alignment software TopHat 2 (v2.1.1) $[46,47]$. The alignment parameters required that each read aligned to the reference's genome sequences at least $75-80 \%$ of the inserted base pairs. Reads with multiple mismatches were excluded from the annotation process, only reads with perfect matches and one mismatch was further analyzed and annotated based on the reference's genome. Transcript level expression for the transcriptome of B. rapa was evaluated using the Cufflinks suite (v2.1.0). A normalized expression level for each $B$. rapa gene model was calculated using the fragments per kilobase of Exon per million fragments mapped (FPKM) normalization method.

\section{Analysis of differentially expressed genes in autotetraploid vs diploid B. rapa \\ To identify differentially expressed genes (DEGs) between the diploid and autotetraploid $B$. rapa DeSeq package from the $\mathrm{R}$ statistical computing environment was used. The resulting probability values were adjusted using the Benjamini and Hochberg method to control the false dis- covery rate [48]. Stringent values of false discovery rate $(F D R) \leq 0.01$ and fold change $(\log 2 \mathrm{FC}) \geq 2$ (p.value 0.05 ) as a threshold were used to identify significant differ- ences in gene expression between the different ploidy.}

\section{Functional classification of DEGs}

For functional annotation of DEGs, bioinformatics tools were employed which included; BLAST similarity searches for gene models search against NCBI nonredundant protein $(\mathrm{Nr})$ database, uniProtKB $\backslash$ Swissprot database, and Kyoto Encyclopedia Genes and Genomes (KEGG) (http://www.genome.jp/kegg/) was used to assign $\mathrm{KO}$ numbers and enrichment pathways to all map transcripts.

\section{Identification of orthologous flowering-related genes in $B$. rapa}

The molecular and functional analysis of flowering has been extensively studied in A. thaliana. Since A. thaliana was considered as a model species for plant systems and the majority of its genes have been functionally annotated, we examined the orthologous floweringrelated gene pairs between $A$. thaliana and B. rapa. Further, for the identified flowering-related genes in $B$. rapa, the BRAD database and chromosome data (v1.5) of Chinese cabbage were employed (http://brassicadb.org/brad/ searchSynteny.php). The flowering-related genes were identified among the ten chromosomes of B. rapa. Furthermore, MCScanX software was used to identify and analyze the flowering-related genes between $B$. rapa and A. thaliana. Circos software was employed to generate the circos figure and Map Chart 2.2 was employed for mapping purposes [49]. Flowering time genes within the photoperiod/circadian clock pathway, meristem response and development, and vernalization and autonomous pathways were examined.

\section{Verification of RNA-seq data}

RNA was extracted from the floral buds of autotetraploid and diploid plants using Trizol reagent (Invitrogen). The qRT-PCR analysis was performed using the Superscript III Platinum SYBR gene qRT-PCR kit (Invitrogen) and QIAGEN ROTOR gene 6000 (QIAGEN) system. Relative expression of interested DEGs was normalized by $\beta$-actin and analyzed using the Delta $2^{-\Delta \Delta C T}$ method. For this analysis, three biological replicates for each sample were run. Gene-specific primers were designed by Primer Premier 5.0 for 9 randomly selected genes. The amplification fragments were between 100 and $200 \mathrm{bp}$. The specificity of all primer pairs was checked with the NCBI primer design tool (NCBI) (Additional file 1: Table S6).

\section{Supplementary Information}

The online version contains supplementary material available at https://doi. org/10.1186/s13578-021-00528-1.

Additional file 1: Table S1. Differentially expressed phytohormones. Table S2. Gene related to organ growth. Table S3. Chromosomal loaction of the Flower time genes among the Subgenomes of B. rapa. Table S4. Differentially expressed genes among the floral transitioning pathways. Table S5. Differentially expressed TF. Table S6. Primers pairs used for qRT-PCR.

Additional file 2: Figure S1. Gene duplication and collinearity analysis among Flower time genes from including B.rapa and A.thaliana. Lines connecting genes depict ortholog pairs diverged from the same ancestor. A1-A10 indicated B.rapa chromosomes, and Ath01-Ath05 displayed A.thaliana chromosomes. Figure S2. RNA-seq results of key genes in the flowering time pathway.

\section{Abbreviations}

DEGs: Differentially expressed genes; GA: Gibberellin; CTK: Cytokinin; BR: Brassinosteroid; SA: Salicylic acid; ABA: Abscisic acid; ETHY: Ethylene; TFs: Transcriptional factors.

\section{Acknowledgments}

This work was financially sponsored by the Program for Science and Technology Innovation Talents in Universities of Henan Province (No. 19HASTIT014), and supported by the National Natural Science Foundation of China (No. 31600995). 


\section{Authors' contributions}

All authors contributed to this manuscript and approved the final manuscript. FW and BT designed and supervised the study, GS and GC participated in design. JB carried out all of the RNA-seq analysis and wrote the manuscript; $J B$ and $Y Y$ participated in the quantitative real-time PCR experiment; JY conducted chromosomal distribution experiment; XW and $X Z$ assisted with pre-treatments of plant materials; ZX assisted in manuscript revision and bioinformatics analysis. All authors read and approved the final manuscript.

\section{Availability of data and materials}

Data availability in NCBI accession: SRP104015.

\section{Ethics approval and consent to participate}

\section{Not applicable.}

\section{Consent of publication}

All authors agree to publish this paper.

\section{Competing interests}

The authors declare that they have no competing interests.

\section{Author details}

${ }^{1}$ School of Life Sciences, Zhengzhou University, Zhengzhou 450001, Henan, China. ${ }^{2}$ Henan International Joint Laboratory of Crop Gene Resources and Improvements, School of Agricultural Sciences, Zhengzhou University, Zhengzhou 450001, Henan, China. ${ }^{3}$ Institute of Horticultural Research, Henan Academy of Agricultural Sciences, Zhengzhou 450002, Henan, China.

Received: 9 July 2020 Accepted: 3 January 2021

Published online: 12 January 2021

\section{References}

1. Soltis PS, Soltis DE. The role of hybridization in plant speciation. Annu Rev Plant Biol. 2009;60:561-88.

2. Sattler MC, Carvalho CR, Clarindo WR. The polyploidy and its key role in plant breeding. Planta. 2016;243(2):281-96.

3. Comai L. The advantages and disadvantages of being polyploid. Nat Rev Genet. 2005;6(11):836-46.

4. Wang X, Wang H, Wang J, Sun R, Wu J, Liu S, et al. The genome of the mesopolyploid crop species Brassica rapa. Nat Genet. 2011;43(10):1035-9.

5. Talebi S, Saharkhiz M, Kermani M, Sharafi Y, Fard F. Effect of different antimitotic agents on polyploid induction of anise hyssop (Agastache foeniculum L.). Caryologia. 2017;70:184-93.

6. Wang J, Tian L, Lee HS, Wei NE, Jiang H, Watson B, et al. Genomewide nonadditive gene regulation in Arabidopsis allotetraploids. Genetics. 2006;172(1):507-17.

7. Weiss-Schneeweiss H, Emadzade K, Jang TS, Schneeweiss GM. Evolutionary consequences, constraints and potential of polyploidy in plants. Cytogenet Genome Res. 2013;140(2-4):137-50.

8. Mu HZ, Liu ZJ, Lin L, Li HY, Jiang J, Liu GF. Transcriptomic analysis of phenotypic changes in birch (Betula platyphylla) autotetraploids. Int J Mol Sci. 2012;13(10):13012-29.

9. Ma Y, Xue H, Zhang L, Zhang F, Ou C, Wang F, et al. Involvement of auxin and brassinosteroid in dwarfism of autotetraploid apple (Malus $\times$ domestica). Sci Rep. 2016;6:26719.

10. Braynen J, Yang Y, Wei F, Cao G, Shi G, Tian B, et al. Transcriptome analysis of floral buds deciphered an irregular course of meiosis in polyploid Brassica rapa. Front Plant Sci. 2017:8:768.

11. Herben T, Suda J, Klimešová J. Polyploid species rely on vegetative reproduction more than diploids: a re-examination of the old hypothesis. Ann Bot. 2017;120(2):341-9.

12. Husband B, Baldwin S, Suda A. The incidence of polyploidy in natural plant populations: major patterns and evolutionary processes, vol. 2. Vienna: Springer; 2013. p. 255-76.

13. Blümel M, Dally $N$, Jung C. Flowering time regulation in crops-what did we learn from Arabidopsis? Curr Opin Biotechnol. 2015;32:121-9.

14. Mayfield D, Chen ZJ, Pires JC. Epigenetic regulation of flowering time in polyploids. Curr Opin Plant Biol. 2011;14(2):174-8.
15. Li X, Yu E, Fan C, Zhang C, Fu T, Zhou Y. Developmental, cytological and transcriptional analysis of autotetraploid Arabidopsis. Planta. 2012;236(2):579-96.

16. Corneillie S, De Storme N, Van Acker R, Fangel JU, De Bruyne M, De Rycke $\mathrm{R}$, et al. Polyploidy affects plant growth and alters cell wall composition. Plant Physiol. 2019;179(1):74-87.

17. Herrmann D, Barre P, Santoni S, Julier B. Association of a CONSTANS-LIKE gene to flowering and height in autotetraploid alfalfa. TAG Theor Appl Genet Theoretische und angewandte Genetik. 2010;121(5):865-76.

18. Song $\mathrm{YH}$, Ito $\mathrm{S}$, Imaizumi T. Flowering time regulation: photoperiod- and temperature-sensing in leaves. Trends Plant Sci. 2013;18(10):575-83.

19. Mouradov A, Cremer F, Coupland G. Control of flowering time: interacting pathways as a basis for diversity. Plant Cell. 2002;14(Suppl):S111-30.

20. Sun TP. Gibberellin metabolism, perception and signaling pathways in Arabidopsis. Arabidopsis Book. 2008;6:e0103.

21. Srikanth A, Schmid M. Regulation of flowering time: all roads lead to Rome. Cell Mol Life Sci CMLS. 2011;68(12):2013-37.

22. Nie S, Li C, Wang Y, Xu L, Muleke EM, Tang M, et al. Transcriptomic Analysis identifies differentially expressed genes (DEGs) associated with bolting and flowering in radish (Raphanus sativus L.). Front Plant Sci. 2016:7:682.

23. Wilson RN, Heckman JW, Somerville CR. Gibberellin Is required for flowering in arabidopsis thaliana under short days. Plant Physiol. 1992;100(1):403-8.

24. Dai Y, Zhang S, Sun X, Li G, Yuan L, Li F, et al. Comparative transcriptome analysis of gene expression and regulatory characteristics associated with different vernalization periods in Brassica rapa. Genes. 2020;11(4):392.

25. Zhai Q, Zhang X, Wu F, Feng H, Deng L, Xu L, et al. Transcriptional mechanism of jasmonate receptor COI1-mediated delay of flowering time in Arabidopsis. Plant Cell. 2015;27(10):2814-28.

26. Del Olmo I, Poza-Viejo L, Piñeiro M, Jarillo JA, Crevillén P. High ambient temperature leads to reduced FT expression and delayed flowering in Brassica rapa via a mechanism associated with H2A.Z dynamics. Plant J Cell Mol Biol. 2019;100(2):343-56.

27. Agliassa C, Narayana R, Bertea CM, Rodgers CT, Maffei ME. Reduction of the geomagnetic field delays Arabidopsis thaliana flowering time through downregulation of flowering-related genes. Bioelectromagnetics. 2018;39(5):361-74.

28. Xi X, Wei K, Gao B, Liu J, Liang J, Cheng F, et al. BrFLC5: a weak regulator of flowering time in Brassica rapa. TAG Theor Appl Genet Theoretische und angewandte Genetik. 2018;131(10):2107-16.

29. Takada S, Akter A, Itabashi E, et al. The role of FRIGIDA and FLOWERING LOCUS C genes in flowering time of Brassica rapa leafy vegetables. Sci Rep. 2019;9:13843.

30. Adamczyk BJ, Lehti-Shiu MD, Fernandez DE. The MADS domain factors AGL15 and AGL18 act redundantly as repressors of the floral transition in Arabidopsis. Plant J Cell Mol Biol. 2007;50(6):1007-19.

31. Duan W, Song X, Liu T, Huang Z, Ren J, Hou X, et al. Genome-wide analysis of the MADS-box gene family in Brassica rapa (Chinese cabbage). Mol Genet Genomics MGG. 2015;290(1):239-55.

32. Liu H, Wang Q, Liu Y, Zhao X, Imaizumi T, Somers DE, et al. Arabidopsis CRY2 and ZTL mediate blue-light regulation of the transcription factor CIB1 by distinct mechanisms. Proc Natl Acad Sci USA. 2013;110(43):17582-7.

33. Krizek BA, Anderson JT. Control of flower size. J Exp Bot. 2013;64(6):1427-37.

34. Jain M, Kaur N, Garg R, Thakur JK, Tyagi AK, Khurana JP. Structure and expression analysis of early auxin-responsive Aux/IAA gene family in rice (Oryza sativa). Funct Integr Genomics. 2006;6(1):47-59.

35. Nambara E, Okamoto M, Tatematsu K, Yano R, Seo M, Kamiya Y. Abscisic acid and the control of seed dormany and germination. Seed Sci Res. 2010;20:55-67.

36. Miransari M, Smith D. Plant hormones and seed germination. Environ Exp Bot. 2013;99:110-21.

37. Voß U, Bishopp A, Farcot E, Bennett MJ. Modelling hormonal response and development. Trends Plant Sci. 2014;19(5):311-9.

38. Wani S, Kumar V, Shriram V, Sah S. Phytohormones and their metabolic engineering for abiotic stress tolerance in crop plants. Crop J. 2016;4:162.

39. Rai MI, Wang X, Thibault DM, Kim HJ, Bombyk MM, Binder BM, et al. The ARGOS gene family functions in a negative feedback loop to desensitize plants to ethylene. BMC Plant Biol. 2015;15(1):157. 
40. Hakata M, Muramatsu M, Nakamura H, Hara N, Kishimoto M, lidaOkada K, et al. Overexpression of TIFY genes promotes plant growth in rice through jasmonate signaling. Biosci Biotechnol Biochem. 2017:81(5):906-13.

41. Anastasiou E, Kenz S, Gerstung M, MacLean D, Timmer J, Fleck C, et al. Control of plant organ size by KLUH/CYP78A5-dependent intercellular signaling. Dev Cell. 2007;13(6):843-56

42. Zhao L, Cai H, Su Z, Wang L, Huang X, Zhang M, et al. KLU suppresses megasporocyte cell fate through SWR1-mediated activation of WRKY28 expression in Arabidopsis. Proc Natl Acad Sci. 2018;115(3):E526-35.

43. Tian Y, Gu H, Fan Z, Shi G, Yuan J, Wei F, et al. Role of a cotton endoreduplication-related gene, GaTOP6B, in response to drought stress. Planta. 2019;249(4):1119-32.

44. Yang Y, Wei F, Braynen J, Wei X, Tian B, Shi G, et al. Cytological and proteomic analyses of floral buds reveal an altered atlas of meiosis in autopolyploid Brassica rapa. Cell Biosci. 2019;9:49.

45. Nicolas SD, Le Mignon G, Eber F, Coriton O, Monod H, Clouet V, et al Homeologous recombination plays a major role in chromosome rearrangements that occur during meiosis of Brassica napus haploids. Genetics. 2007;175(2):487-503.

46. Cheng F, Liu S, Wu J, Fang L, Sun S, Liu B, et al. BRAD, the genetics and genomics database for Brassica plants. BMC Plant Biol. 2011;11:136.

47. Trapnell C, Roberts A, Goff L, Pertea G, Kim D, Kelley DR, et al. Differential gene and transcript expression analysis of RNA-seq experiments with TopHat and Cufflinks. Nat Protoc. 2012;7(3):562-78.

48. Anders $\mathrm{S}$, Huber W. Differential expression analysis for sequence count data. Genome Biol. 2010;11(10):R106.

49. Krzywinski M, Schein J, Birol I, Connors J, Gascoyne R, Horsman D, et al. Circos: an information aesthetic for comparative genomics. Genome Res. 2009;19(9):1639-45.

\section{Publisher's Note}

Springer Nature remains neutral with regard to jurisdictional claims in published maps and institutional affiliations.
Ready to submit your research? Choose BMC and benefit from:

- fast, convenient online submission

- thorough peer review by experienced researchers in your field

- rapid publication on acceptance

- support for research data, including large and complex data types

- gold Open Access which fosters wider collaboration and increased citations

- maximum visibility for your research: over $100 \mathrm{M}$ website views per year

At BMC, research is always in progress.

Learn more biomedcentral.com/submissions 